\title{
ABCD Autumn Meeting Abstracts 2018
}

\author{
The 2018 ABCD autumn meeting took place on 8th and 9th November at BMA House in \\ London. One of the top scoring abstract is included here and the remaining can be found \\ online at www.bjd-abcd.com. Some abstracts could not be published as they have already \\ been published elsewhere.
}

The Paediatric Diabetes QI Collaborative: a national pilot of 10 paediatric diabetes units in England

Peng M, Muszynska K, Eardley S, Hopper N, Woodhead T, Campbell $F$

Royal College of Paediatrics and Child Health, HQIP, National Children \& Young People's Diabetes Network

Inspired by the National Diabetes QI Collaborative in Sweden between 2010 and 2012, the Royal College of Paediatrics and Child Health conducted a pilot QI collaborative in 2017 with 10 paediatric diabetes multidisciplinary teams (MDTs) from across England. All members of each MDT were expected to attend a 9-month programme of training consisting of two residential weekends and two one-day events, with additional lunchtime webinars for team champions and access to a secure online platform to share resources and ideas with each other between events. The training encompassed improving outcomes in complex systems, the PDSA cycle, the value of time-ordering measurement, reliability and human factors, the values and behaviours of a high performing team, process mapping and fishbone analysis as a starter for change, and the engagement of children and young people. Initial run-chart data of outcome measures collected during the collaborative show a $5-10 \%$ reduction in mean $\mathrm{HbA} 1 \mathrm{c}$ pre- and post-pilot. Qualitative feedback from the teams at the end of the programme reported a noticeable change in team function and they were either likely or highly likely to continue their QI activities beyond the collaborative. All 10 teams rated their experience of participating in the QI programme as either very or extremely useful. As part of the new National Children and Young People's Diabetes Quality Programme, two further national waves of the QI collaborative started in October 2018 with subsequent regional waves of single-day training events commencing in March 2019, recruiting over $85 \%$ of all paediatric diabetes units in England and Wales in the next 3 years.

Bridging the gap: piloting integrated clinical health psychology and young adult diabetes services in Wrexham Stewart R, Griffiths $C$, Stanaway $S$

Betsi Cadwaladr University Health Board

This paper details the service design and outcomes of a 2-year pilot project involving a clinical psychologist working as an embedded team member within a young adult type 1 diabetes service. Key components of the pilot included the introduction of psychosocial screening measures for all patients, fully integrated multidisciplinary clinics, a rapid assessment and intervention model for patients aged 18-30, complex case risk formulation and staff training on psychological issues. Psychosocial screening data on 101 patients indicated that $23(24 \%)$ experienced clinical levels of diabetes distress (primarily emotional burden). Screening on a cohort of 93 patients showed that 17 (18\%) patients experienced clinical levels of depression, and screening on a cohort of 60 patients indicated that 12 (20\%) experienced clinically concerning levels of eating problems. Pre and post self-report measures indicate that patients who attended for therapy appointments with the psychologist reported significant improvements in depression $(t(29)=2.64, p=0.0066)$ and diabetes distress $(t(34)=2.46, p=0.01)$. Data from unscheduled care usage show that, during the duration of the pilot project, the diabetic ketoacidosis (DKA) admission rate for the age group covered by the project reduced by over $40 \%$ and the number of repeat DKA admissions (admissions for individuals with at least one previous DKA admission within the previous year) dropped to zero.

A not so common cause of diabetic ketoacidosis in a type 2 diabetic patient

Shah V, Zac-Varghese S, George S

East and North Hertfordshire NHS Trust

Diabetic ketoacidosis (DKA) is a major acute complication in type 1 diabetes mellitus that requires immediate treatment and correction. However, uncommonly, DKA can also occur in type 2 diabetes mellitus. We report a case of an 83-year-old patient with type 2 diabetes with DKA due to an uncommon trigger. The patient was diagnosed 10 years ago and was on biphasic insulin. She presented to the emergency department feeling lethargic with polyuria and polydipsia. She was tachycardic and tachypnoeic but normotensive and apyrexic with no obvious focus of infection. Her capillary blood glucose was $26 \mathrm{mmol} / \mathrm{L}$, ketones $4.8 \mathrm{mmol} / \mathrm{L}$ and $\mathrm{pH} 7.19$ with bicarbonate of 14 . She met the criteria for DKA and was started on the appropriate management pathway for DKA. Despite resolution of DKA on day 2 and return to her normal biphasic insulin regime, she remained tachypnoeic and tachycardic and lethargic. Subsequent investigation of her thyroid status showed that her thyroid stimulating hormone was $<0.03$ with free T4 level of $64.1 \mu \mathrm{g} / \mathrm{L}$. She was also thyroid stimulating hormone receptor (TSH-R) antibody positive, confirming Grave's thyroid disease. She was then started on propranolol and carbimazole on day 5 with good resolution of her symptoms in 24 hours. On day 7 she was medically stable and referred on for physical rehabilitation on discharge. This case highlights the importance of identifying promptly the triggers for DKA - in this case thyrotoxicosis - as appropriate management for both is required to prevent mortality and morbidity. Hyperthyroidism is not a commonly known cause of DKA and we recommend routinely testing for thyroid state in unexplained DKA. 
Physician perception and clinical practice regarding use of SGLT2 inhibitors in patients with foot ulcer disease Bassett JK, Ahmed SH

Countess of Chester Hospital NHS Foundation Trust

Introduction: Canagliflozin, the first licensed sodium-glucose cotransporter 2 (SGLT-2) inhibitor in the EU, has been linked to an increased risk of leg and foot amputations in the CANVAS/CANVAS-R trials. Some believe that other members of this class, dapagliflozin and empagliflozin, are innocent bystanders maligned by this side effect. This study seeks to elucidate physician perception of the use of dapagliflozin/empagliflozin in light of the EMEA communication on canagliflozin.

Methods: A questionnaire was sent to 70 physicians including consultants and specialist trainees within the UK exploring their prescription practice of dapagliflozin/empagliflozin (not canagliflozin) in the context of incident and historical foot ulcer disease.

Results: Of 53 clinicians surveyed, 25\% would not start an SGLT-2 inhibitor under any circumstances, $45 \%$ would consider dapagliflozin/empagliflozin regardless of aetiology of previous foot ulcers and a further 30\% would start if ulcers were of neuropathic origin. For patients with incident foot ulcers, 54\% would stop them, $55 \%$ of which would restart them if the incident ulcer was confirmed neuropathic in origin. Of the clinicians who did continue them with incident ulcers (46\%), 38\% would stop if the ulcers were confirmed to be ischaemic or neuro-ischaemic.

Discussion: Our results illustrate a diversity of views regarding dapagliflozin/empagliflozin prescription amongst physicians in the context of foot ulcer disease. A recent announcement from the DECLARE-TIMI 58 trial group has indicated positive outcomes in terms of cardiovascular safety for dapagliflozin. The EMPA-REG OUTCOME trial likewise has shown a reduction in cardiovascular risk with empagliflozin. Safety and efficacy studies for dapagliflozin/empagliflozin have not shown an increased risk of amputation. Should patients be denied the benefits of glycaemic control and lowered cardiovascular risk that these SGLT-2 inhibitors confer?

Gestational diabetes: IADPSG versus NICE diagnostic dilemmas Cooke B, Thiraviaraj A

Altnagelvin Hospital

Introduction: Gestational diabetes (GDM) is testing the resources and service capacity of district general hospitals around the UK. Despite the clear evidence that glucose intolerance in pregnancy has short and long term consequences for both mother and baby, there is still a lack of overall consensus for diagnosis. ${ }^{1}$ Our service uses the outcome-based International Association of Diabetes and Pregnancy Study Groups (IADPSG) criteria. ${ }^{2}$

Methods: We performed a retrospective audit of women diagnosed with gestational diabetes ( $n=383$, mean age 32.5 years) attending antenatal services within our Trust in Northern Ireland. This was mainly a white Caucasian population. Data were collected on baseline characteristics and outcomes including delivery, birth weight and neonatal complications. We also identified the subgroup of our patients diagnosed as GDM with IADPSG criteria who would have been 'non-diagnostic' by NICE diagnostic criteria (ie, fasting plasma glucose $<5.6 \mathrm{mmol} / \mathrm{L} \pm 2$-hour glucose $<7.8 \mathrm{mmol} / \mathrm{L}$ ). ${ }^{3}$

Results: Of our total 383 patients, 181 would have missed detection with NICE criteria (47\%). These patients were significantly heavier at booking (almost $7 \mathrm{~kg}$ ), which was sustained until delivery. Of this subgroup, 80 women required metformin and 26 required treatment with insulin ( \pm metformin) to achieve NICE targets. This was comparable to the subgroup classified as GDM by NICE. There was no significant difference between outcomes in both.

Conclusion: Although comparable outcomes were achieved with our subgroups of patients with GDM, it was notable that $>50 \%$ of those 'NICE non-diagnostic' women required medication to achieve blood glucose control.

\section{References}

1. Duran A, Saenz S, Torrejon M, et al. Introduction of IADPSG criteria for the screening and diagnosis of gestational diabetes mellitus results in improved pregnancy outcomes at a lower cost in a large cohort of pregnant women: the St Carlos Gestational Diabetes Study. Diabetes Care 2014:37:2442-50. https://doi.org/10.2337/dc14-0179

2. Metzger BE, Gabbe SG, Persson B, et al; International Association of Diabetes and Pregnancy Study Groups Consensus Panel. International Association of Diabetes and Pregnancy Study Groups recommendations on the diagnosis and classification of hyperglycaemia in pregnancy. Diabetes Care 2010;33:676-82. https://doi.org/10.2337/ dc09-1848

3. National Institute for Health and Care Excellence. Diabetes in pregnancy: management from preconception to the postnatal period. NICE guideline [NG3]. 2015. https://www.nice.org.uk/guidance/ng3

Detecting insulin sensitivity changes for individuals with type 1 diabetes

\section{Lewis DM, Street TJ, Leibrand S, Phatak SS}

Arizona State University

Background: A method to calculate changes in insulin needs was developed in the OpenAPS (Open Source Artificial Pancreas System) community. Individuals have natural fluctuations in insulin needs, but excessive periods of sensitivity or resistance may indicate ongoing physiological trends and therefore impact management of type 1 diabetes.

Methods: Autosens analyses each continuous glucose monitoring (CGM) data point for 24 hours, comparing observed change to expected impact from insulin. Autosens calculates the deviation for the median of the last 8 and 24 hours of CGM data points and determines the sensitivity ratio (SR) required to neutralise the median deviation. Autosens was run retrospectively to obtain an hourly SR value (first calculated SR every hour) for $(\mathrm{N}=1) * 16$ individuals using OpenAPS, with $M=5,393$ data points (range 922-20,473). A SR of $>1.0$ indicates resistance and $<1.0$ indicates sensitivity. Histograms were created to visualise SR for each participant. Mean \pm SD SR was calculated and those falling beyond $\pm 10 \%$ of 1.0 were classified as being resistant and sensitive respectively.

Results: Mean SR for 12 individuals fell within $\pm 10 \%$ of 1.0 . One individual tended towards sensitivity $(0.79 \pm 16)$ and three individuals tended towards resistance $(1.2 \pm 0.28 ; 1.33 \pm 0.30 ; 1.41 \pm 0.37)$.

Conclusion: Such idiographic visualisation of SR can be useful for detecting overall patterns of sensitivity/resistance potentially unaccounted for by the user's pump settings.

\section{Mitochondrial diabetes: don't ignore clinical clues! Witczak JK, Ravindran $R$, Adlan M, Premawardhana $L$ Department of Medicine, Ysbyty Ystrad Fawr Hospital, Hengoed}

Background: Mitochondrial diabetes has an incidence of $1 \%$, is maternally inherited and leads to gradual beta cell failure. A high index of clinical suspicion and a strong family history may help establish the diagnosis. 
Case presentation: An 18-year-old obese (BMI 30) female with recent onset of diabetes mellitus was referred because of an atypical presentation and a strong family history of diabetes (mother, grandmother and great-grandmother). She was asymptomatic at presentation (no osmotic symptoms, tiredness or weight loss). However, fasting plasma glucose was $7.2 \mathrm{~mol} / \mathrm{L}$ and an oral glucose tolerance test confirmed diabetes. She was ketone free. Essential hypertension (on perindopril) and 'fibromyalgia' (intense muscle pains and tenderness) had been diagnosed previously. Genetic screening established that she was negative for HNF and glucokinase mutations but she had the m.3243A>G mutation confirming the clinical suspicion of mitochondrial diabetes. Her $\mathrm{HbA}_{1 \mathrm{c}}$ fell from 63 to $48 \mathrm{mmol} / \mathrm{mol}$ on metformin (MF) and diet alone. However, MF was withdrawn because of the risk of lactic acidosis, but her $\mathrm{HbA}_{1 c}$ deteriorated on sitagliptin and MF was restarted as she wished to avoid sulphonylureas and insulin because of the risk of weight gain.

Discussion: (1) Diabetes mellitus is part of the clinical picture in subjects presenting with mitochondrial mutations. (2) Some have deafness, myopathy, lactic acidosis and heart disease associated with diabetes. (3) An atypical presentation (young and asymptomatic in our subject) coupled with a strong maternal family history should alert clinicians to the diagnosis. (4) Our patient had the commonest mutation identified with this syndrome. (5) Genetic counselling and family case detection should be undertaken and involvement of other systems should be excluded. (6) Beta cell dysfunction is progressive in this condition and many progress to require insulin at some stage. 